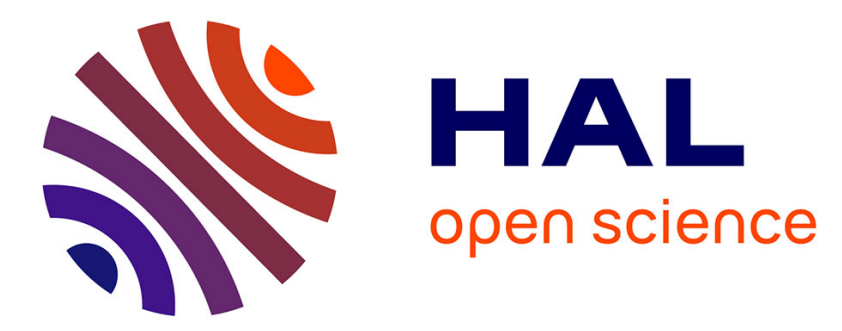

\title{
Interfaces in micromorphic materials: Wave transmission and reflection with numerical simulations
} Arkadi Berezovski, Ivan Giorgio, Alessandro Della Corte

\section{To cite this version:}

Arkadi Berezovski, Ivan Giorgio, Alessandro Della Corte. Interfaces in micromorphic materials: Wave transmission and reflection with numerical simulations. Mathematics and Mechanics of Solids, 2016, 21 (1), pp.37-51. 10.1177/1081286515572244 . hal-01194376

\section{HAL Id: hal-01194376 https://hal.science/hal-01194376}

Submitted on 6 Sep 2015

HAL is a multi-disciplinary open access archive for the deposit and dissemination of scientific research documents, whether they are published or not. The documents may come from teaching and research institutions in France or abroad, or from public or private research centers.
L'archive ouverte pluridisciplinaire HAL, est destinée au dépôt et à la diffusion de documents scientifiques de niveau recherche, publiés ou non, émanant des établissements d'enseignement et de recherche français ou étrangers, des laboratoires publics ou privés. 


\title{
Interfaces in micromorphic materials: wave transmission and reflection with numerical simulations
}

\author{
Arkadi Berezovski; Ivan Giorgio, Alessandro Della Corte $\ddagger$
}

October 22, 2014

\begin{abstract}
Reflection and transmission of elastic waves at the interface between two distinct micromorphic media are considered in the one-dimensional setting. Dual internal variable approach is used for the description of the microstructure influence on the global motion. It is shown that reflection and transition coefficients for plane waves depend on the coupling between macro- and micro-motions as well as on the choice of the microstructural interaction at the interface. Numerical simulations exhibiting results with promising technological implications are shown.
\end{abstract}

\section{Introduction}

The technical possibility to accurately constructing and controlling materials at the micro- and nano-scale is today rapidly increasing. Theoretical predictions about the behavior of micromorphic materials, as well as soundly based numerical analysis on the same subject, are therefore particularly relevant scientific topics at the moment [1-6, e.g.]. In the present work, we will focus on transmission and reflection of elastic waves.

Reflection and transmission of elastic waves is studied well [7, 8] even in the case of inhomogeneous materials [9-12, e.g.]. The underlying jump relations at interfaces [13] have been extended to the case of phase mixtures [14], stationary and moving material interfaces in thermoelastic solids, [15-17], and even imperfect interfaces [18]. Anyway, all the extensions mentioned above relate to the description of wave propagation at the macrolevel. The investigation of the influence of a microstructure on the reflection and transmission of elastic waves is usually restricted by micropolar materials and only recently [19,20] is applied to second-gradient materials. Since the second-gradient elasticity is only a particular case of the generalized continua theory [21], it is worth to consider a more broad case, which represents one of the most critical topics in today's continuum mechanics, i.e. micromorphic materials.

The micromorphic elasticity has been introduced long ago [22, 23], and wave propagation in micromorphic materials has been studied as well [24]. However, wave reflection and transmission attracted much less attention up to now, due to the number of additional material parameters. However, recent theoretical [25], experimental [26], and computational [27] results open the way for practical applications of the micromorphic theory [28, 29, e.g.].

The internal variables can be thought as kinematical descriptors, whose physical meaning is given by the phenomenology to describe which the model is intended. One well established example of materials which can be suitably described by a dual internal variable micromorphic model is given by dual scale porosity, i.e. materials characterized by two porous structures at different length scales. In this case, the change in volume fraction with respect to the reference configuration is taken as a common kinematical descriptor [30]. An example can be also provided by relaxed linear micromorphic continuum in which one considers a microstrain tensor and micro-dislocations [5]. Another example can be given by an unsatured soil in which a porous structure is filled by a liquid and a vapor, whose respective porosities constitute the two kinematical descriptors relative to the microstructure [31].

In what follows, reflection and transmission of elastic waves at the boundary between two distinct micromorphic media is analyzed in the one-dimensional setting. The study is performed in the framework of the material formulation of continuum mechanics (Sect. 2) incorporating internal variables (Sect. 3). The dual internal variables concept is used for the description of micromorphic media (Sect. 4). The formulation of jump relations (Sect. 5) and a constitutive model (Sect. 6) complete the background. It is shown (Sect. 7) that even in this simplified description the reflection and transmission coefficients for plane

\footnotetext{
${ }^{*}$ Centre for Nonlinear Studies, Institute of Cybernetics at Tallinn University of Technology, Tallinn, Estonia.

${ }^{\dagger}$ Dep. of Mechanical and Aerospace Engineering, SAPIENZA Università di Roma, via Eudossiana 18, 00184 Rome, Italy; Int. Research Center for the Mathematics and Mechanics of Complex Systems (MeMoCS), Palazzo Caetani, 04012 Cisterna di Latina, Italy.

${ }^{\ddagger}$ Dep. of Mechanical and Aerospace Engineering, SAPIENZA Università di Roma, SAPIENZA Università di Roma, via Eudossiana 18, 00184 Rome, Italy; Int. Research Center for the Mathematics and Mechanics of Complex Systems (MeMoCS), Palazzo Caetani, 04012 Cisterna di Latina, Italy.
} 
waves depend on the coupling between micro- and macro-motion. Numerical simulations are exhibited (Sect. 8) in some relevant cases.

\section{Balance laws in the material formulation}

One-dimensional motion of thermoelastic conductors of heat is governed by local balance laws for linear momentum and energy (no body forces)

$$
\begin{gathered}
\left(\rho_{0} v\right)_{t}-\sigma_{x}=0, \\
\left(\rho_{0} v^{2} / 2+E\right)_{t}-(\sigma v-Q)_{x}=0
\end{gathered}
$$

and by the second law of thermodynamics

$$
S_{t}+(Q / \theta+K)_{x} \geq 0
$$

Here $t$ is time, $\rho_{0}$ is the matter density, $v=u_{t}$ is the physical velocity, $\sigma$ is the Cauchy stress, $E$ is the internal energy per unit volume, $S$ is the entropy per unit volume, $\theta$ is temperature, $Q$ is the material heat flux, and $K$ is the 'extra entropy flux' which can be assumed, in most cases, to be vanishing [32]. Indices denote differentiation with respect to the corresponding variable.

The formulated balance laws do not 'feel' any internal structure. The influence of a microstructure can be described by means of the introduction of additional internal degrees of freedom (like in [22, 23]) or internal variables of state [33]. Here we concentrate on the internal variables approach. These internal variables are governed by their own evolution equations. To derive evolution equations for internal variables, we turn to the material formulation of continuum mechanics [34].

The canonical energy equation is obtained by introducing the free energy per unit volume $W:=E-S \theta$ and taking into account balance of linear momentum (1)

$$
(S \theta)_{t}+Q_{x}=h^{i n t}, \quad h^{i n t}:=\sigma \varepsilon_{t}-W_{t},
$$

where the right-hand side of Eq. (4) 1 is formally an internal heat source [35].

In the case of non-zero extra entropy flux, the second law of thermodynamics gives then

$$
-\left(W_{t}+S \theta_{t}\right)+\sigma \varepsilon_{t}+(\theta K)_{x}-(Q / \theta+K) \theta_{x} \geq 0,
$$

where $\varepsilon=u_{x}$ is the one-dimensional strain measure.

Multiplying Eq. (1) by $u_{x}$ we then check that Eq. (1) yields the following material balance of momentum (cf. [34])

$$
P_{t}-b_{x}=f^{i n t}+f^{i n h},
$$

where the material momentum $P$, the material Eshelby stress $b$, the material inhomogeneity force $f^{i n h}$, and the material internal force $f^{\text {int }}$ are defined by [34]

$$
\begin{gathered}
P:=-\rho_{0} u_{t} u_{x}, \quad b:=-\left(\rho_{0} v^{2} / 2-W+\sigma \varepsilon\right), \\
f^{i n h}:=\left(\frac{1}{2} v^{2}\right) \frac{\partial \rho_{0}}{\partial x}-\left.\frac{\partial W}{\partial x}\right|_{\text {expl }}, \quad f^{i n t}:=\sigma u_{x x}-\left.\frac{\partial W}{\partial x}\right|_{i m p l} .
\end{gathered}
$$

Here the subscript notations expl and impl mean, respectively, the derivative keeping the fields fixed (and thus extracting the explicit dependence on $x$ ), and taking the derivative only through the fields present in the function. Canonical equations for energy and momentum (4) and (6) are written in the general case, without a specification of the free energy $W$.

\section{Single internal variable}

Now we are ready to introduce internal variables which supposedly describe the influence of a microstructure on the overall macroscopic motion of a body. For the sake of simplicity, we start with the single internal variable theory [35, 36].

Let $\psi$ be the internal variable of state which we associate with the distributed effect of the microstructure. Then the free energy density $W$ is taken as a sufficiently regular function of the strain, temperature, the internal variable, and its space gradient [35]

$$
W=\bar{W}\left(u_{x}, \theta, \psi, \psi_{x}\right)
$$

The equations of state (in a sense, mere definition of the partial derivatives of the free energy) are given by

$$
\sigma=\frac{\partial \bar{W}}{\partial u_{x}}, \quad S=-\frac{\partial \bar{W}}{\partial \theta}, \quad \tau:=-\frac{\partial \bar{W}}{\partial \psi} \quad \eta:=-\frac{\partial \bar{W}}{\partial \psi_{x}} .
$$


The presence of internal variables leads to a modification of the governing balance laws. To be consistent, let us consider first dissipation inequality (5), which is represented as

$$
\Phi=\tau \psi+\eta\left(\psi_{x}\right)_{t}-(Q / \theta+K) \theta_{x}+(\theta K)_{x} \geq 0 .
$$

Rearranging the dissipation inequality we arrive at

$$
\Phi=\left(\tau-\eta_{x}\right) \psi_{t}-(Q / \theta+K) \theta_{x}+\left(\eta \psi_{t}+\theta K\right)_{x} \geq 0 .
$$

Following [37], we select the 'extra' entropy flux in order to eliminate the divergence term in Eq. (12)

$$
K=-\theta^{-1} \eta \psi_{t} .
$$

Such a choice results in the following canonical equations of momentum and energy [35, 36]

$$
\begin{gathered}
P_{t}-\widetilde{b}_{x}=f^{t h}+\widetilde{f}^{i n t r}, \\
(S \theta)_{t}+\widetilde{Q}_{x}=h^{t h}+\widetilde{h}^{i n t r} .
\end{gathered}
$$

Here the introduced thermal source terms involve only temperature

$$
f^{t h}:=S \theta_{x}, \quad h^{t h}:=S \theta_{t},
$$

whereas 'intrinsic' source terms are determined by the internal variable

$$
\widetilde{f}^{\text {intr }}:=\widetilde{\tau} \psi_{x}, \quad \widetilde{h}^{\text {intr }}:=\widetilde{\tau} \psi_{t} .
$$

The modified Eshelby stress tensor

$$
\widetilde{b}=-\left(\rho_{0} v^{2} / 2-W+\sigma u_{x}-\eta \psi_{x}\right),
$$

includes all terms presenting gradients, with the material gradient of $\psi$ playing a role parallel to that of the deformation gradient. Remaining modifications are defined as follows $[35,36]$

$$
\widetilde{S}=\theta^{-1} \widetilde{Q}, \quad \widetilde{Q}=Q-\eta \psi_{t}, \quad \widetilde{\tau} \equiv-\frac{\delta \bar{W}}{\delta \psi}:=-\left(\frac{\partial \bar{W}}{\partial \psi}-\frac{\partial}{\partial x}\left(\frac{\partial \bar{W}}{\partial \psi_{x}}\right)\right)=\tau-\eta_{x},
$$

and the dissipation inequality is represented as

$$
\Phi=\widetilde{\tau} \psi_{t}-\left(\frac{Q-\eta \psi_{t}}{\theta}\right) \theta_{x} \geq 0
$$

The main advantage of the use of the material formulation of thermomechanics is the possibility to derive an evolution equation for the introduced internal variable consistent with the second law. Dissipation inequality (20) is clearly decomposed into intrinsic and thermal parts. The standard choice to require non-negativity of the intrinsic part of the dissipation inequality (20)

$$
\widetilde{h}^{i n t r}=\widetilde{\tau} \psi_{t}=\left(\tau-\eta_{x}\right) \psi_{t} \geq 0,
$$

leads to the evolution equation for the internal variable in the form

$$
k \psi_{t}=\tau-\eta_{x}, \quad k \geq 0 .
$$

This is a parabolic reaction-diffusion-like equation, as usual for the standard dissipative internal variable $[33,35,36]$. In the non-dissipative case $(k=0)$, the latter is reduced to

$$
\tau-\eta_{x}=0,
$$

thus resulting in a second-gradient theory. 


\section{Dual internal variables}

The single internal variable theory cannot describe, in many cases, the behavior of micromorphic bodies. Fortunately, this can be done by means of its generalization [38-40]. The generalization of the internal variable theory on the case of two internal variables is straightforward. Let us consider the free energy density $W$ as a function of two internal variables, $\psi, \varphi$, and their space derivatives

$$
W=\bar{W}\left(u_{x}, \psi, \psi_{x}, \varphi, \varphi_{x}\right) .
$$

The equations of state are given by

$$
\sigma:=\frac{\partial \bar{W}}{\partial u_{x}}, \quad \tau:=-\frac{\partial \bar{W}}{\partial \psi}, \quad \eta:=-\frac{\partial \bar{W}}{\partial \psi_{x}}, \quad \xi:=-\frac{\partial \bar{W}}{\partial \varphi}, \quad \zeta:=-\frac{\partial \bar{W}}{\partial \varphi_{x}} .
$$

We include into consideration the non-zero extra entropy flux according to the case of one internal variable

$$
K=-\theta^{-1} \eta \psi_{t}-\theta^{-1} \zeta \xi_{t} .
$$

The canonical equations of momentum and energy keep their form

$$
\begin{gathered}
P_{t}-\widetilde{b}_{x}=f^{t h}+\widetilde{f}^{i n t r}, \\
(S \theta)_{t}+\widetilde{Q}_{x}=h^{t h}+\widetilde{h}^{i n t r},
\end{gathered}
$$

with the modified Eshelby stress tensor

$$
\widetilde{b}=-\left(\frac{1}{2} \rho_{0} v^{2}-W+\sigma u_{x}-\eta \psi_{x}-\zeta \varphi_{x}\right),
$$

and intrinsic source terms

$$
\begin{aligned}
& \widetilde{f}^{\text {intr }}:=\left(\tau-\eta_{x}\right) \psi_{x}+\left(\xi-\zeta_{x}\right) \varphi_{x}, \\
& \widetilde{h}^{\text {intr }}:=\left(\tau-\eta_{x}\right) \psi_{t}+\left(\xi-\zeta_{x}\right) \varphi_{t} .
\end{aligned}
$$

The entropy flux is modified similarly to the case of single internal variable

$$
\widetilde{S}=\theta^{-1} \widetilde{Q}, \quad \widetilde{Q}=Q-\eta \psi_{t}-\zeta \varphi_{t} .
$$

The corresponding dissipation inequality

$$
\Phi=\left(\tau-\eta_{x}\right) \psi_{t}+\left(\xi-\zeta_{x}\right) \varphi_{t}-\left(\frac{Q-\eta \psi_{t}-\zeta \varphi_{t}}{\theta}\right) \theta_{x} \geq 0 .
$$

in the isothermal case is reduced to

$$
\Phi=\left(\tau-\eta_{x}\right) \psi_{t}+\left(\xi-\zeta_{x}\right) \varphi_{t} \geq 0
$$

It is easy to see that in the non-dissipative case $\left(\widetilde{h}^{\text {intr }}=0\right)$ dissipation inequality (34) can by satisfied by the choice

$$
\psi_{t}=L\left(\xi-\zeta_{x}\right), \quad \varphi_{t}=-L\left(\tau-\eta_{x}\right),
$$

where $L$ is an arbitrary coefficient. The latter two evolution equations express the coupling between internal variables: one internal variable is driven by another one and vice versa.

\section{Jump relations}

To be able to analyze the reflection and transmission of elastic waves at an interface between two micromorphic media, we need to formulate jump relations for all fields at this interface. Let $\llbracket A \rrbracket=A^{+}-A^{-}$, the jump of a discontinuous field $A$ across an interface denoted by $\Sigma$. Let $V$ the material velocity of $\Sigma$. The interface $\Sigma$ is considered as homothermal (no jump in temperature) and coherent (no defects such as dislocations). Consequently, we have the following continuity conditions [34]:

$$
\llbracket \theta \rrbracket=0 \quad \text { and } \quad \llbracket V \rrbracket=0 \quad \text { at } \quad \Sigma .
$$


Jump relations associated with bulk equations (1) and (2) that are strict conservation laws, read in the absence of body forces

$$
\begin{gathered}
V_{\Sigma} \llbracket \rho_{0} v \rrbracket+\llbracket \sigma \rrbracket=0, \\
V_{\Sigma} \llbracket \rho_{0} v^{2} / 2+E \rrbracket+\llbracket \sigma v-Q \rrbracket=0,
\end{gathered}
$$

where $V_{\Sigma}$ is the speed of the points of $\Sigma$.

The same rule can be applied to bulk equations (27) and (28), which are not strict conservation laws, if we formally add unknown source terms, i.e., we a priori write the following two jump relations [34]:

$$
\begin{aligned}
& V_{\Sigma} \llbracket P \rrbracket+\llbracket \widetilde{b} \rrbracket+f_{\Sigma}=0, \\
& V_{\Sigma} \llbracket S \rrbracket-\llbracket \widetilde{Q} / \theta \rrbracket=\sigma_{\Sigma} .
\end{aligned}
$$

The second law of thermodynamics at $\Sigma$ reads

$$
\sigma_{\Sigma} \geq 0
$$

Here $\sigma_{\Sigma}$ is the entropy production and $f_{\Sigma}$ is the driving (configurational) force acting at the interface.

\subsection{Stationary interface}

If the interface $\Sigma$ does not move, then $V_{\Sigma}=0$, and the jump relations are reduced to

$$
\begin{gathered}
\llbracket \sigma \rrbracket=0, \\
\llbracket \sigma v-Q \rrbracket=0, \\
\sigma_{\Sigma}=-\llbracket \widetilde{Q} / \theta \rrbracket, \\
f_{\Sigma}=-\llbracket \widetilde{b} \rrbracket,
\end{gathered}
$$

In the isothermal non-dissipative case the entropy production is zero which results in

$$
\sigma_{\Sigma}=0 \quad \Leftrightarrow \quad \llbracket \widetilde{Q} \rrbracket=0 \quad \Leftrightarrow \quad \llbracket Q-\eta \psi_{t}-\zeta \varphi_{t} \rrbracket=0 .
$$

It follows from the latter relation and Eq. (43) that

$$
\llbracket \sigma v+\eta \psi_{t}+\zeta \varphi_{t} \rrbracket=0
$$

Other jump relations remain unchanged.

\section{Constitutive model}

To get the dispersive wave equation, let us consider the following form for the quadratic free energy density for a Mindlin-type microstructure model in the one-dimensional case [41]

$$
W=\frac{1}{2}(\lambda+2 \mu) \varepsilon^{2}+A \varepsilon \psi+\frac{B}{2} \psi^{2}+\frac{C}{2} \psi_{x}^{2}+\frac{D}{2} \varphi^{2} .
$$

Accordingly, the one-dimensional Cauchy stress is

$$
\sigma \equiv \frac{\partial W}{\partial \varepsilon}=(\lambda+2 \mu) \varepsilon+A \psi,
$$

and the corresponding microstress $\eta$ has the form

$$
\eta \equiv-\frac{\partial W}{\partial \psi_{x}}=-C \psi_{x}
$$

For the interaction force $\tau$ we have

$$
\tau \equiv-\frac{\partial W}{\partial \psi}=-A \varepsilon-B \psi
$$


Equations of motion include the balance of linear momentum in the absence of body forces

$$
\rho_{0} u_{t t}=\sigma_{x}
$$

which is represented in terms of deformations as

$$
\rho_{0} u_{t t}=(\lambda+2 \mu) \varepsilon_{x}+A \psi_{x},
$$

and evolution equation for the microdeformation following from Eq. (35)

$$
M \psi_{t t}=\tau-\eta_{x}
$$

or

$$
M \psi_{t t}=C \psi_{x x}-A \varepsilon-B \psi
$$

with $M=1 / L^{2} D$.

It can be checked that the energy flux $H$ in the case of one-dimensional micromorphic bodies is represented as

$$
H=(\lambda+2 \mu) \varepsilon v+A \psi v+C \psi_{x} \psi_{t}
$$

\subsection{Single dispersive wave equation}

The governing equations mentioned above can be represented as a single higher-order equation. In fact, the space derivative of $\psi$ can be determined from Eq. (55)

$$
B \psi_{x}=C \psi_{x x x}-A \varepsilon_{x}-M \psi_{t t x} .
$$

Higher derivatives of $\psi$ in the right-hand side of Eq. (57) are calculated by means of Eq. (53)

$$
\begin{gathered}
A \psi_{t t x}=\rho_{0} u_{t t t t}-(\lambda+2 \mu) \varepsilon_{t t x}, \\
A \psi_{x x x}=\rho_{0} u_{x x t t}-(\lambda+2 \mu) \varepsilon_{x x x} .
\end{gathered}
$$

Inserting obtained results into Eq. (53), we have

$$
\rho_{0} u_{t t}-(\lambda+2 \mu) u_{x x}=-\frac{M}{B}\left(\rho_{0} u_{t t}-(\lambda+2 \mu) u_{x x}\right)_{t t}+\frac{C}{B}\left(\rho_{0} u_{t t}-(\lambda+2 \mu) u_{x x}\right)_{x x}-\frac{A^{2}}{B} u_{x x} .
$$

This is the most general one-dimensional Mindlin-type microstructure model [42].

\section{Plane waves}

To show the influence of a microstructure on the reflection and transmission of elastic waves it is sufficient to restrict ourselves to plane waves. In this case, the solution of Eq. (60) is supposed in the form

$$
u=\alpha e^{i(k x-\omega t)}
$$

where $\omega$ is the angular frequency and $k$ is the wave number.

Then the dispersion relation follows in the form

$$
\rho_{0} \omega^{2}-(\lambda+2 \mu) k^{2}=\frac{M}{B} \omega^{2}\left(\rho_{0} \omega^{2}-(\lambda+2 \mu) k^{2}\right)-\frac{C}{B} k^{2}\left(\rho_{0} \omega^{2}-(\lambda+2 \mu) k^{2}\right)-\frac{A^{2}}{B} k^{2} .
$$

This dispersion relation is studied deeply in $[42,43]$. It possesses two real solutions corresponding to propagative waves and two imaginary solutions for the so-called standing (or evanescent) waves (c.f. [19]). 


\subsection{System of equations}

For further analysis, it is convenient to represent governing equations (53) and (55) in the form of the system of first-order equations. In fact, the balance of linear momentum (53) can be rewritten in terms of velocity as

$$
\rho_{0} v_{t}=(\lambda+2 \mu) \varepsilon_{x}+A \psi_{x},
$$

accompanying by the compatibility condition

$$
\varepsilon_{t}=v_{x}
$$

Introducing the time rate and gradient of the microdeformation, $w$ and $\varkappa$, respectively, by

$$
\psi_{t}=w, \quad \psi_{x}=\varkappa,
$$

one can rewrite the evolution equation for the primary internal variable (55) as

$$
M w_{t}=C \varkappa_{x}-A \varepsilon-B \psi
$$

together with the corresponding compatibility conditions following from Eq. (65)

$$
\varkappa_{t}=w_{x} .
$$

\subsection{Jump relations at a stationary interface}

Jump relations corresponding to the formulated system of equations are

$$
\begin{aligned}
\llbracket(\lambda+2 \mu) \varepsilon & +A \psi \rrbracket=0, \\
\llbracket v \rrbracket & =0, \\
\llbracket C \varkappa \rrbracket & =-g, \\
\llbracket w \rrbracket & =0 .
\end{aligned}
$$

Here $g$ corresponds to the source term for the microstructural jump relation. Conditions (68)-(71) are the most general relations at a stationary interface in the case of non-dissipative isothermal deformation of micromorphic media. They become uncoupled if $A=0$, and determine complete continuity if, in addition, $g=0$. The choice of the source term $g$ is dictated by the microstructure interaction at the boundary between two different micromorphic media. For additional information about contact interactions in more general cases see e.g. [44]. The coupling parameter $A$ determines the interaction between macromotion and microstructure not only in the jump relations, but also in the governing equations.

\subsection{Reflection and transmission at a stationary interface}

Considering the plane wave motion

$$
u=\alpha e^{i(k x-\omega t)}, \quad \psi=i k \beta e^{i(k x-\omega t)},
$$

we have for the macrostrain and velocity

$$
\varepsilon=i k \alpha e^{i(k x-\omega t)}, \quad v=-i \omega \alpha e^{i(k x-\omega t)},
$$

and for the gradient and the time rate of microdeformation

$$
\varkappa=-k^{2} \beta e^{i(k x-\omega t)}, \quad w=k \omega \beta e^{i(k x-\omega t)} .
$$

Denoting the reflection coefficients by $R_{\alpha}$ and $R_{\beta}$, respectively, and the transmission coefficients by $T_{\alpha}$ and $T_{\beta}$, accordingly, we have, due to jump relation (68)

$$
\begin{aligned}
(\lambda+2 \mu)_{I} k_{I} \alpha+A_{I} k_{I} \beta & +(\lambda+2 \mu)_{I} k_{I} R_{\alpha} \alpha+R_{\beta} A_{I} k_{I} \beta= \\
& =(\lambda+2 \mu)_{T} k_{T} T_{\alpha} \alpha+T_{\beta} A_{T} k_{T} \beta,
\end{aligned}
$$

where subscripts $I$ and $T$ correspond to media for incident and transmitted wave, respectively. Correspondingly, it follows from jump relation (69) that

$$
1-R_{\alpha}=T_{\alpha}
$$


The reflection and transmission coefficients for the microstructure yield from remaining jump relations (70) and (71). In the simplest case with $g=0$ we have

$$
\begin{gathered}
k_{I}^{2} C_{I}+k_{I}^{2} C_{I} R_{\beta}=k_{T}^{2} C_{T} T_{\beta}, \\
k_{I}-k_{I} R_{\beta}=k_{T} T_{\beta} .
\end{gathered}
$$

Multiplying the latter equation by $k_{I} C_{I}$, we obtain

$$
k_{I}^{2} C_{I}-k_{I}^{2} C_{I} R_{\beta}=k_{T} k_{I} C_{I} T_{\beta} .
$$

Summing up Eqs. (77) and (79) we arrive at

$$
2 k_{I}^{2} C_{I}=k_{T} k_{I} C_{I} T_{\beta}+k_{T}^{2} C_{T} T_{\beta},
$$

which results in the explicit expression for the transmission coefficients for the microstructure

$$
T_{\beta}=\frac{2 k_{I}^{2} C_{I}}{C_{I} k_{T} k_{I}+k_{T}^{2} C_{T}} .
$$

Then the reflection coefficient is retrieved by means of Eq. (78)

$$
R_{\beta}=-\frac{k_{I}^{2} C_{I}-k_{I} k_{T} C_{T}}{k_{I}^{2} C_{I}+k_{I} k_{T} C_{T}} .
$$

Substituting the values of $T_{\beta}$ and $R_{\beta}$ into Eq. (75), we can calculate the values of macroscopic reflection and transmission coefficients taking into account Eq. (76). Indeed, Eq. (75) can be represented in the form

$$
(\lambda+2 \mu)_{I} k_{I}+(\lambda+2 \mu)_{I} k_{I} R_{\alpha}-(\lambda+2 \mu)_{T} k_{T} T_{\alpha}=\varepsilon M,
$$

where $\varepsilon=\beta / \alpha$ is the ratio of amplitudes of micro- and macro-waves, and $M$ is the influence of reflection and transmission at micro-level, namely,

$$
M=T_{\beta} A_{T} k_{T}-R_{\beta} A_{I} k_{I}-A_{I} k_{I} .
$$

For non-zero values of $\varepsilon$ we have then

$$
T_{\alpha}=\frac{2(\lambda+2 \mu)_{I} k_{I}-\varepsilon M}{(\lambda+2 \mu)_{I} k_{I}+(\lambda+2 \mu)_{T} k_{T}}, \quad R_{\alpha}=\frac{(\lambda+2 \mu)_{T} k_{T}-(\lambda+2 \mu)_{I} k_{I}+\varepsilon M}{(\lambda+2 \mu)_{I} k_{I}+(\lambda+2 \mu)_{T} k_{T}} .
$$

If $\varepsilon=0$ (no influence of microstucture), reflection and transmission coefficients are reduced to classical values

$$
T_{\alpha}=\frac{2(\lambda+2 \mu)_{I} k_{I}}{(\lambda+2 \mu)_{I} k_{I}+(\lambda+2 \mu)_{T} k_{T}}, \quad R_{\alpha}=\frac{(\lambda+2 \mu)_{T} k_{T}-(\lambda+2 \mu)_{I} k_{I}}{(\lambda+2 \mu)_{I} k_{I}+(\lambda+2 \mu)_{T} k_{T}} .
$$

Remembering that $k=\omega / c$, where $c=\sqrt{(\lambda+2 \mu) / \rho}$, we can represent the latter expressions as

$$
T_{\alpha}=\frac{2 \rho_{I} c_{I}}{\rho_{I} c_{I}+\rho_{T} c_{T}}, \quad R_{\alpha}=\frac{\rho_{T} c_{T}-\rho_{I} c_{I}}{\rho_{I} c_{I}+\rho_{T} c_{T}}
$$

which is exactly the common form of reflection and transmission coefficients for homogeneous media $[7,8]$.

\section{Numerical simulations}

Numerical simulations of wave transmission and reflection at the interface between two different micromorphic materials are employed to demonstrate the influence of microstructure on wave propagation. The main aim is to show the effects of changes in the micro-macro coupling on the characteristics of the reflected and transmitted waves. All the numerical simulations were performed using the commercial software COMSOL Multiphysics ${ }^{\circledR}$. We used a FEM method with a uniform mesh with step $L / 10^{3}$ and a time step $3.16 \times 10^{-5} \mathrm{~s}$.

It should be noted that for numerical simulations we considered the following explicit form for the energy $W$ :

$$
W=\frac{1}{2}(\lambda+2 \mu) \varepsilon^{2}+\frac{A_{1}}{2}(\varepsilon-\psi)^{2}+\frac{A_{2}}{2}(\varepsilon-\varphi)^{2}+\frac{B_{1}}{2} \psi^{2}+\frac{B_{2}}{2} \varphi^{2}+\frac{C_{1}}{2} \psi_{x}^{2}+\frac{C_{2}}{2} \varphi_{x}^{2}+\frac{D_{1}}{2}(\psi-\varphi)^{2}+\frac{D_{2}}{2}\left(\psi_{x}-\varphi_{x}\right)^{2}
$$

The values of microstructural material parameters are presented in Table 1. 


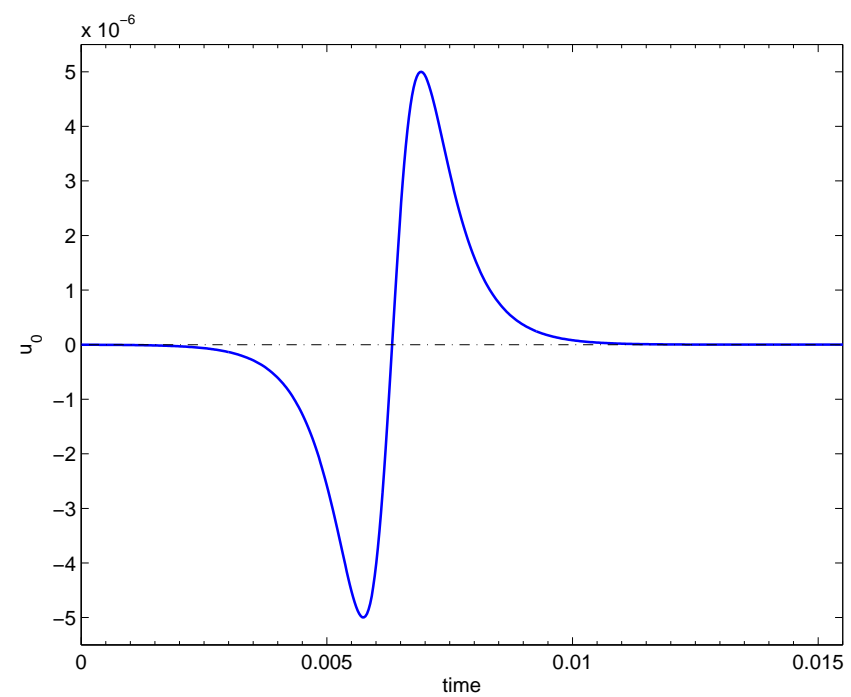

Figure 1: Displacement perturbation.

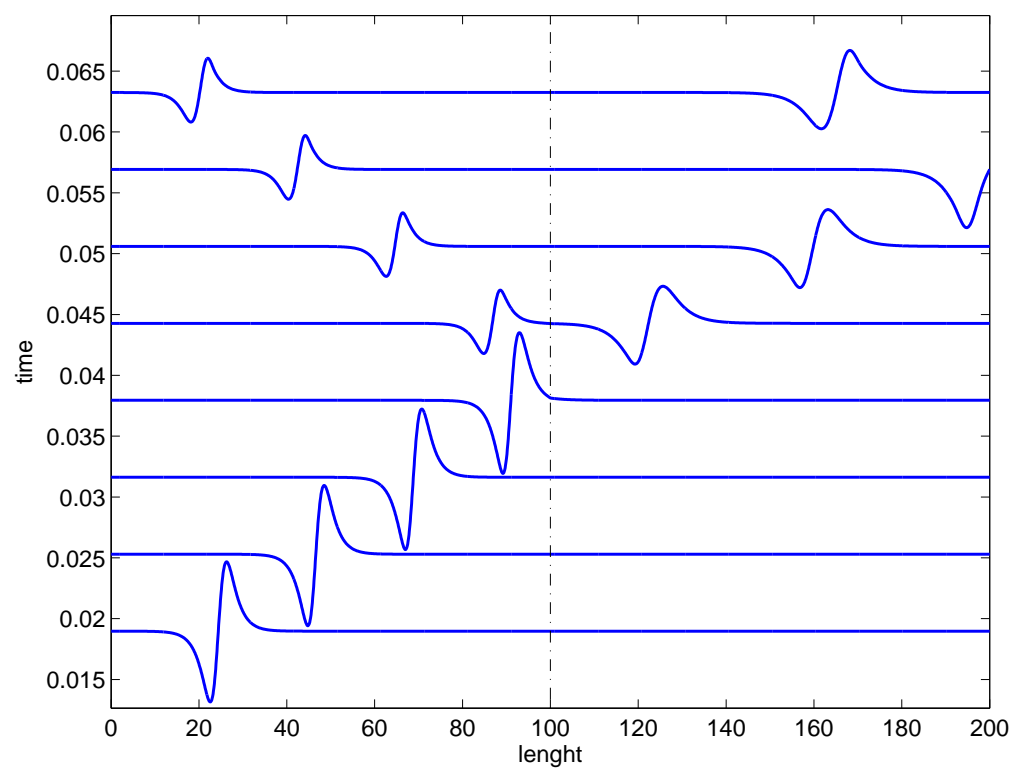

Figure 2: Wave propagation in a two-bars system. 
Table 1: Reference constitutive parameters for the two materials employed.

\begin{tabular}{|c|c|c|c|c|c|c|c|c|c|}
\hline & $K_{\varepsilon}$ & $A_{1}$ & $A_{2}$ & $B_{1}$ & $B_{2}$ & $C_{1}$ & $C_{2}$ & $D_{1}$ & $D_{2}$ \\
\hline Mat. 1 & $18 \mathrm{e} 9 \mathrm{~Pa}$ & $1.8 \mathrm{e} 8 \mathrm{~Pa}$ & $1.8 \mathrm{e} 9 \mathrm{~Pa}$ & $1.8 \mathrm{e} 11 \mathrm{~Pa}$ & 7.2e11 Pa & $1.8 \mathrm{e} 6 \mathrm{~N}$ & $1.8 \mathrm{e} 6 \mathrm{~N}$ & $9.0 \mathrm{e} 8 \mathrm{~Pa}$ & $1.8 \mathrm{e} 6 \mathrm{~N}$ \\
\hline Mat. 2 & $80 \mathrm{e} 9 \mathrm{~Pa}$ & 8.0e8 Pa & 8.0e9 $\mathrm{Pa}$ & 8.0e11 Pa & 3.2e12 $\mathrm{Pa}$ & $8.0 \mathrm{e} 6 \mathrm{~N}$ & $8.0 \mathrm{e} 6 \mathrm{~N}$ & $4.0 \mathrm{e} 9 \mathrm{~Pa}$ & $8.0 \mathrm{e} 6 \mathrm{~N}$ \\
\hline
\end{tabular}

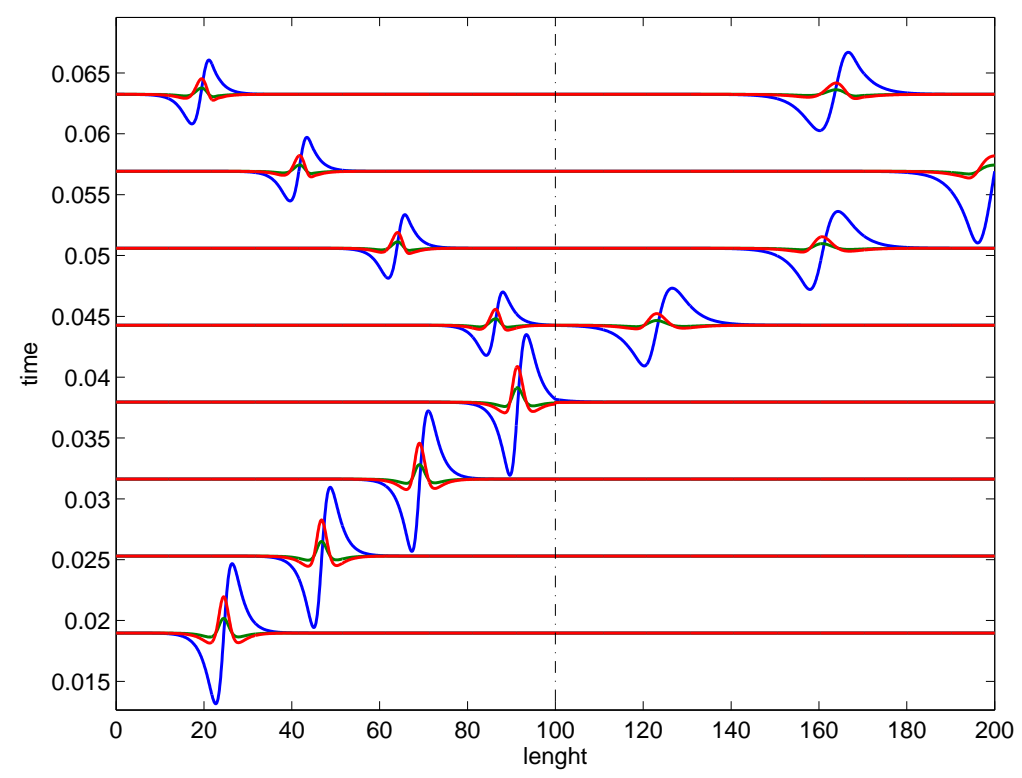

Figure 3: Wave propagation in a two-bars system with a weak micro-macro coupling. (Displacement $u$, blue line; $\psi$, green line; $\varphi$, red line). The scale factor of $\psi$ and $\varphi$ is 200 times larger than that of $u$.

The first simulation is considered as a 'reference' case. We considered here a junction of two simple homogeneous bars (whose length is $L=100 \mathrm{~m}$ ) of different elastic materials without microstructure, the stiffer one being on the right. The loading is applied at the left boundary in the form of a perturbation of the displacement

$$
u(0, t)=U_{0} \operatorname{sech}\left[\alpha\left(t-t_{\text {ref }}\right)\right] \tanh \left[\alpha\left(t-t_{\text {ref }}\right)\right]
$$

(plotted in Fig. 1) and the right boundary is fixed $(u(L, t)=0)$. The amplitude of the perturbation is set as $U_{0}=10^{-5} \mathrm{~m}$, $\alpha=1500 \mathrm{1} / \mathrm{s}$, and the reference time $t_{\text {ref }}=1 / 158 \mathrm{~s}$ to obtain a wave length of about $20 \mathrm{~m}$ in the 'reference' case.

At the interface between the two materials, we imposed continuity in $u$. The results, displaying ordinary transmission and reflection phenomena, are presented in Fig. 2. The difference in the velocity of wave propagation in distinct materials is clearly observed.

In the second simulation, we considered the same problem adding two microstructural descriptors, $\psi$ and $\varphi$ for each material. The ratios between the bulk stiffness $K_{\varepsilon}=\lambda+2 \mu$ and material parameters related to the micro-structure are the same for the two materials (see Table 1). At the interface, continuity in $u, \psi$ and $\varphi$ is imposed. Since we used a FEM approach to perform the simulations, no other interface conditions were needed. Setting weak micro-macro coupling terms, we observed that reflection and transmission not much different from the ones observed in the reference case (with no microstructure), as one can see in Fig. 3. This is, of course, an expected result.

Instead, higher values of the coupling terms result in a dramatically different behavior in Fig. 4, with a marked micro-macro energy transfer (in this case we set for the Mat. 2: $A_{1}=4.0 \mathrm{e} 11 \mathrm{~Pa}$ and $A_{2}=8.0 \mathrm{e} 11 \mathrm{~Pa}$ ).

In the next simulation, we considered the case of a small inclusion (whose length is $L_{\text {inc }}=20 \mathrm{~m}$ ) embedded into a material. For the sake of comparison, here we again start with the case with no micro-structure (see Fig. 5). The material parameters of the bar and the inclusion correspond respectively to Mat. 1 and Mat. 2 in Table 1.

Within this system we imposed two different sets of values for the coupling terms between the bar and the inclusion. In the first case, we considered a lower value for the coupling terms in the micromorphic material relative to the micromorphic inclusion. As one can see in corresponding Fig. 6, small amplitudes for the reflected waves are observed (we set for the Mat. 1: $A_{1}=9.0 \mathrm{e} 11 \mathrm{~Pa}$ and $\left.A_{2}=3.6 \mathrm{e} 11 \mathrm{~Pa}\right)$. 


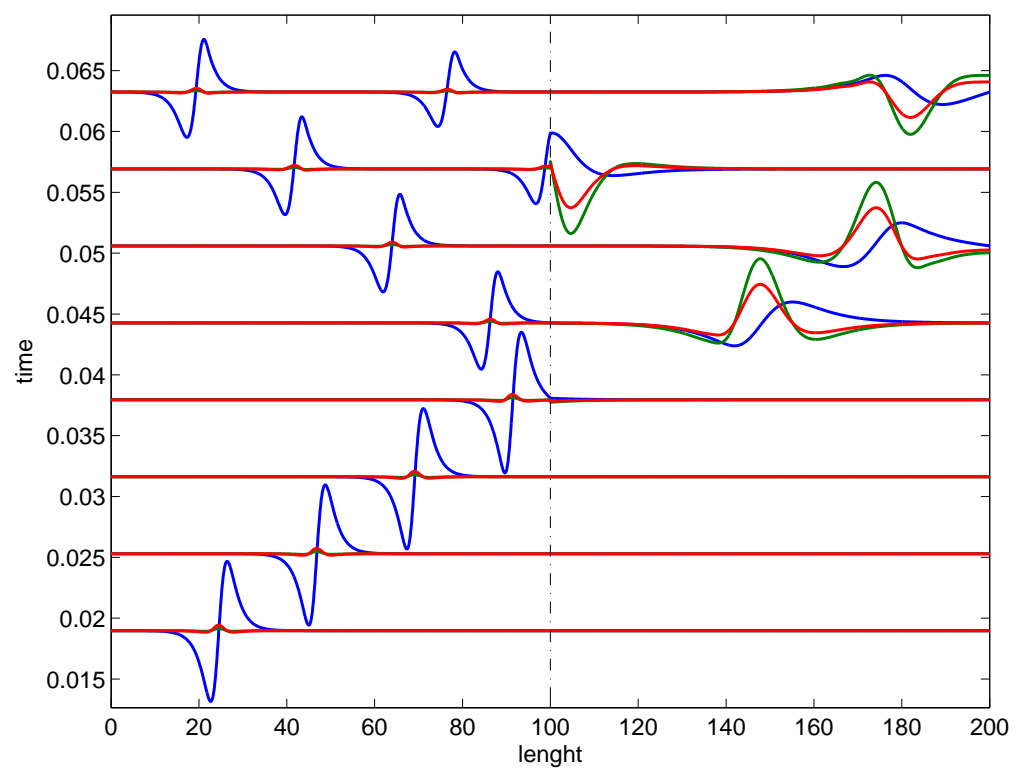

Figure 4: Wave propagation in a two-bars system with a strong micro-macro coupling on the right side. (Displacement $u$, blue line; $\psi$, green line; $\varphi$, red line). The scale factor of $\psi$ and $\varphi$ is 36.69 times larger than that of $u$.

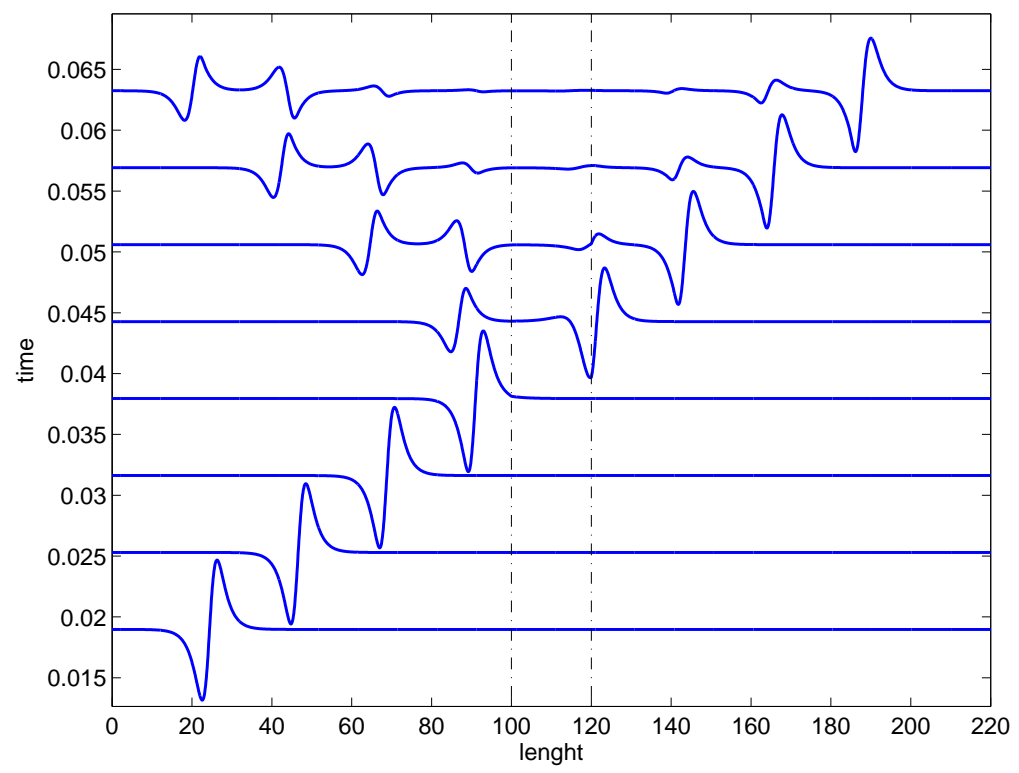

Figure 5: Wave propagation in a bar with an inclusion at the center. 


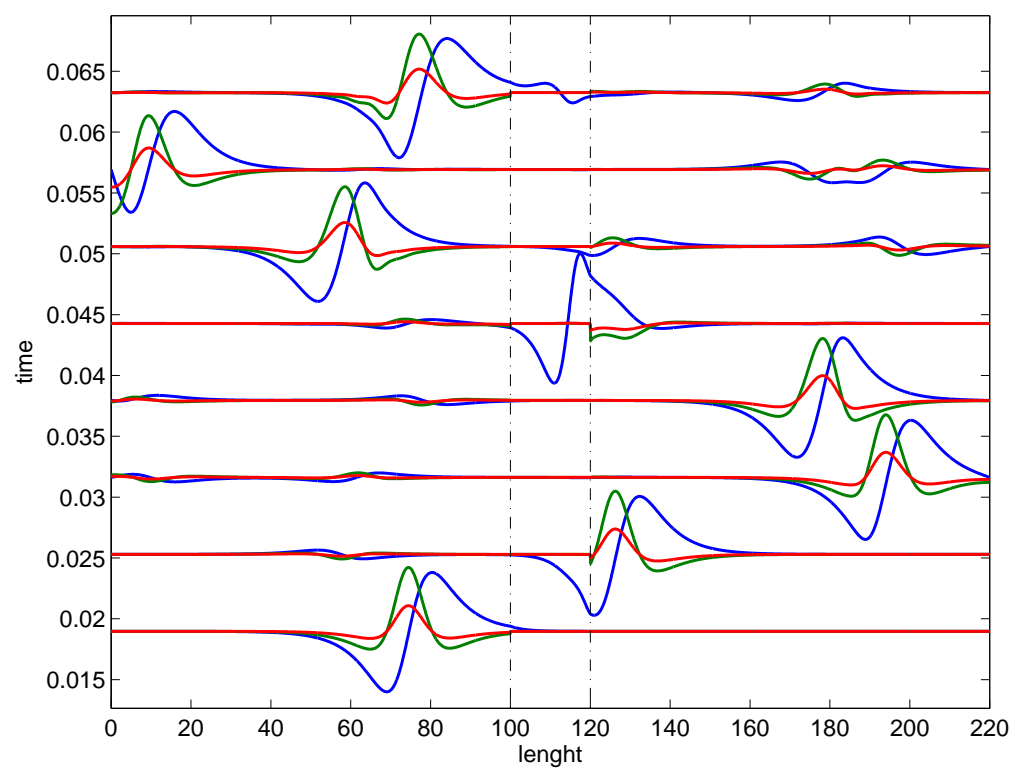

Figure 6: Wave propagation in a bar with an inclusion characterized by weak micro-macro coupling. (Displacement $u$, blue line; $\psi$, green line; $\varphi$, red line). The scale factor of $\psi$ and $\varphi$ is 4.53 times larger than that of $u$.

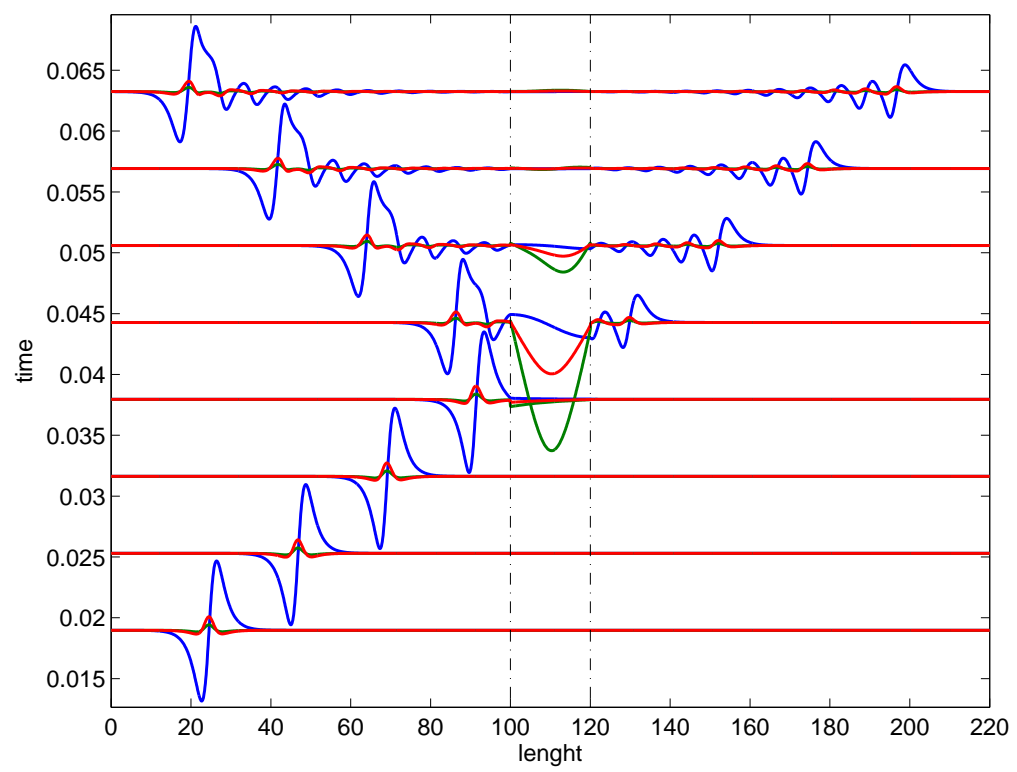

Figure 7: Wave propagation in a bar with an inclusion characterized by strong micro-macro coupling. (Displacement $u$, blue line; $\psi$, green line; $\varphi$ red line). The scale factor of $\psi$ and $\varphi$ is 82.27 times larger than that of $u$. 
In the subsequent simulation, we considered instead the 'mirror' case, i.e. a higher value for the coupling terms for the micromorphic inclusion (we set for the Mat. 2: $A_{1}=4.0 \mathrm{e} 12 \mathrm{~Pa}$ and $A_{2}=1.6 \mathrm{e} 12 \mathrm{~Pa}$ ). In this case, we obtained significant effects which were not easy to forecast without numerical computing. In particular, a high (negative) peak is observable in the value of the microstructural kinematic descriptor $\psi$ (see Fig. 7). In this simulation we observed very different behaviors as a consequence of differences in the coupling terms. A dispersion of the energy related to this peak in the 'macro-energy', dependent on the displacement $u$, is afterwards clearly observable (a similar behavior, though in a different context, was shown in [45]). This phenomenon gives the possibility to 'entrap' peaks of energy in the microstructure and could open the path to novel possibilities for the design of damping filters.

Let us conclude our description of the numerical results with a brief discussion about the future possibility to extend the previously mentioned ideas up to real applications. First of all let us note that, even though our numerical analyses were limited to the one-dimensional case, they still represent a sensible basis for the theoretical viability of these technological possibilities. Of course, in order to proceed towards real-life applications, 2D and ultimately 3D numerical simulations, whose computational needs are much more demanding, are required. It is indeed possible to refine the numerical formulation in view of performing such simulations on higher-dimensional domains within reasonable computational costs, as studied e.g. in $[46,47]$. Another feature to be taken into account in view of actual application of the models developed herein to real structures is that the imperfections present in real materials may cause or influence global or local instabilities. The literature in the field is very wide; to stay within the theoretical framework developed in the present work, the reader is referred to the analysis of one-dimensional systems developed e.g. in $[48,49]$.

\section{Conclusions}

The simplest possible model of the wave reflection and transmission at the boundary between two distinct micromorphic media is considered in the paper. It is shown how the reflection and transmission depends on the microstructural parameters. In principle, the choice of the microstructure interaction term $g$ should play its own role. It is clear that the obtained values of reflection and transmission coefficients generalize classical results for homogeneous materials.

As already said in the introduction, theoretical prediction and numerical analysis of the qualitative behavior of wave propagation in micromorphic materials is becoming more and more technologically relevant as the accuracy in the process of constructing and controlling the microstructure is rapidly increasing. What is by now significant, indeed, is the possibility to manipulate reflection and transmission coefficients by means of microstructure, since it can be controlled or constructed artificially in a sufficiently reliable way. Numerical simulations show, in particular, the role of micro-macro coupling terms in the dispersion, which could be exploited in a number of potential applications.

\section{Acknowledgements}

The research was supported by the EU through the European Regional Development Fund, by the Estonian Science Foundation (grant No. 8702), and by the Estonian Research Council grant PUT 434 (A.B.).

\section{References}

[1] Hirschberger CB, Steinmann P. Modelling of Microstructured Materials with Micromorphic Continuum Approaches. In: Recent Developments and Innovative Applications in Computational Mechanics. Springer; 2011. p. 191-198.

[2] Grillo A, Federico S, Wittum G. Growth, mass transfer, and remodeling in fiber-reinforced, multi-constituent materials. International Journal of Non-Linear Mechanics. 2012;47(2):388-401.

[3] Forest S. Micromorphic media. In: Generalized Continua from the Theory to Engineering Applications. Springer; 2013. p. 249-300.

[4] Altenbach H, Eremeyev VA. Direct approach-based analysis of plates composed of functionally graded materials. Archive of Applied Mechanics. 2008;78(10):775-794.

[5] Neff P, Ghiba ID, Madeo A, Placidi L, Rosi G. A unifying perspective: the relaxed linear micromorphic continuum. Continuum Mechanics and Thermodynamics. 2013;p. 1-43.

[6] Dingreville R, Robbins J, Voth TE. Wave propagation and dispersion in elasto-plastic microstructured materials. International Journal of Solids and Structures. 2014;51(11):2226-2237. 
[7] Achenbach J. Wave Propagation in Elastic Solids. Elsevier; 1984.

[8] Graff KF. Wave Motion in Elastic Solids. Courier Dover Publications; 1975.

[9] Keller HB. Propagation of stress discontinuities in inhomogeneous elastic media. SIAM Review. 1964;6:356-382.

[10] Cooper HF. Propagation of one-dimensional waves in inhomogeneous elastic media. Siam Review. 1967;9:671-679.

[11] Quiligotti S, Maugin GA, dell'Isola F. Wave motions in unbounded poroelastic solids infused with compressible fluids. Zeitschrift für angewandte Mathematik und Physik ZAMP. 2002;53(6):1110-1138.

[12] Wang Y, Hao H. Modelling of guided wave propagation with spectral element: Application in structural engineering. Applied Mechanics and Materials. 2014;553:687-692.

[13] Hill R. Discontinuity relations in mechanics of solids. Progress in Solid Mechanics. 1961;2(72):245-276.

[14] Morland L, Sellers S. Multiphase mixtures and singular surfaces. International Journal of Non-Linear Mechanics. 2001;36(1):131-146.

[15] Maugin GA. On shock waves and phase-transition fronts in continua. ARI-An International Journal for Physical and Engineering Sciences. 1998;50(3):141-150.

[16] Steinmann P, Häsner O. On material interfaces in thermomechanical solids. Archive of Applied Mechanics. 2005;75(1):31-41.

[17] Irschik H. On rational treatments of the general laws of balance and jump, with emphasis on configurational formulations. Acta Mechanica. 2007;194(1-4):11-32.

[18] Maugin GA, Rousseau M. Wave-quasi-particle dualism in the transmission-reflection problem for elastic waves. Journal of Theoretical and Applied Mechanics. 2012;50:797-805.

[19] dell'Isola F, Madeo A, Placidi L. Linear plane wave propagation and normal transmission and reflection at discontinuity surfaces in second gradient 3D continua. ZAMM-Journal of Applied Mathematics and Mechanics/Zeitschrift für Angewandte Mathematik und Mechanik. 2012;92(1):52-71.

[20] Placidi L, Rosi G, Giorgio I, Madeo A. Reflection and transmission of plane waves at surfaces carrying material properties and embedded in second-gradient materials. Mathematics and Mechanics of Solids. 2013;19(5):555-578.

[21] Eringen AC. Microcontinuum Field Theories. Vol. I. Springer; 1999.

[22] Mindlin RD. Micro-structure in linear elasticity. Archive for Rational Mechanics and Analysis. 1964;16:51-78.

[23] Eringen AC, Suhubi E. Nonlinear theory of simple micro-elastic solids-I. International Journal of Engineering Science. 1964;2(2):189-203.

[24] Cowin SC, Nunziato JW. Linear elastic materials with voids. Journal of Elasticity. 1983;13(2):125-147.

[25] Chen Y, Lee JD, Eskandarian A. Atomistic viewpoint of the applicability of microcontinuum theories. International Journal of Solids and Structures. 2004;41(8):2085-2097.

[26] Maranganti R, Sharma P. A novel atomistic approach to determine strain-gradient elasticity constants: Tabulation and comparison for various metals, semiconductors, silica, polymers and the (Ir) relevance for nanotechnologies. Journal of the Mechanics and Physics of Solids. 2007;55(9):1823-1852.

[27] Fish J, Kuznetsov S. From Homogenization to Generalized Continua. International Journal for Computational Methods in Engineering Science and Mechanics. 2012;13(2):77-87.

[28] Wang X, Lee JD. Micromorphic theory: a gateway to nano world. International Journal of Smart and Nano Materials. 2010;1(2):115-135.

[29] dell'Isola F, Rosa L, Woźniak C. A micro-structured continuum modelling compacting fluid-saturated grounds: The effects of pore-size scale parameter. Acta Mechanica. 1998;127(1-4):165-182.

[30] Ieşan D. Method of potentials in elastostatics of solids with double porosity. International Journal of Engineering Science. 2014;. 
[31] Madeo A, dell'Isola F, Darve F. A continuum model for deformable, second gradient porous media partially saturated with compressible fluids. Journal of the Mechanics and Physics of Solids. 2013;61(11):2196-2211.

[32] Maugin GA. On canonical equations of continuum thermomechanics. Mechanics Research Communications. 2006;33(5):705-710.

[33] Maugin GA, Muschik W. Thermodynamics with internal variables Part I. General concepts. Journal of Non-Equilibrium Thermodynamics. 1994;19(3):217-249.

[34] Maugin GA. Material Inhomogeneities in Elasticity. CRC Press; 1993.

[35] Maugin GA. On the thermomechanics of continuous media with diffusion and/or weak nonlocality. Archive of Applied Mechanics. 2006;75:723-738.

[36] Maugin GA. Configurational Forces: Thermomechanics, Physics, Mathematics, and Numerics. CRC Press; 2010.

[37] Maugin GA. Infernal variables and dissipative structures. Journal of Non-Equilibrium Thermodynamics. 1990;15:173192.

[38] Ván P, Berezovski A, Engelbrecht J. Internal variables and dynamic degrees of freedom. Journal of Non-Equilibrium Thermodynamics. 2008;33:235-254.

[39] Berezovski A, Engelbrecht J, Maugin GA. Generalized thermomechanics with dual internal variables. Archive of Applied Mechanics. 2011;81:229-240.

[40] Engelbrecht J, Berezovski A. Internal structures and internal variables in solids. Journal of Mechanics of Materials and Structures. 2013;7:983-996.

[41] Berezovski A, Engelbrecht J, Maugin GA. One-dimensional microstructure dynamics. In: Mechanics of Microstructured Solids. Springer; 2009. p. 21-28.

[42] Berezovski A, Engelbrecht J, Berezovski M. Waves in microstructured solids: A unified viewpoint of modeling. Acta Mechanica. 2011;220:349-363.

[43] Engelbrecht J, Berezovski A, Pastrone F, Braun M. Waves in microstructured materials and dispersion. Philosophical Magazine. 2005;85:4127-4141.

[44] dell'Isola F, Seppecher P, Madeo A. How contact interactions may depend on the shape of Cauchy cuts in Nth gradient continua: Approach "à la D'Alembert". Zeitschrift für Angewandte Mathematik und Physik. 2012;63(6):1119-1141.

[45] Rosi G, Giorgio I, Eremeyev VA. Propagation of linear compression waves through plane interfacial layers and mass adsorption in second gradient fluids. ZAMM-Journal of Applied Mathematics and Mechanics/Zeitschrift für Angewandte Mathematik und Mechanik. 2013;93(12):914-927.

[46] Cazzani A, Malagù M, Turco E. Isogeometric analysis of plane-curved beams. Mathematics and Mechanics of Solids. 2014;p. 1081286514531265.

[47] Greco L, Cuomo M. An implicit G1 multi patch B-spline interpolation for Kirchhoff-Love space rod. Computer Methods in Applied Mechanics and Engineering. 2014;269:173-197.

[48] Luongo A, Zulli D, Piccardo G. A linear curved-beam model for the analysis of galloping in suspended cables. Journal of Mechanics of Materials and Structures. 2007;2(4):675-694.

[49] Luongo A, Piccardo G. A continuous approach to the aeroelastic stability of suspended cables in 1:2 internal resonance. Journal of Vibration and Control. 2008;14(1-2):135-157. 